\title{
The Effect of Differences in Methodology Among Some Recent Applications of Shearing Quotients
}

\author{
Doug M. Boyer, ${ }^{1 *}$ Julia Winchester, ${ }^{2}$ and Richard F. Kay ${ }^{1}$ \\ ${ }^{1}$ Department of Evolutionary Anthropology, Duke University, Durham, NC 27708 \\ ${ }^{2}$ Interdepartmental Program in Anthropological Sciences, Stony Brook University, Stony Brook, NY 11790
}

\begin{abstract}
KEY WORDS Platyrrhini; mastication; structural carbohydrate; PGLS; discriminant function analysis; Strepsirrhini; dietary ecology; adaptation
\end{abstract}

\begin{abstract}
A shearing quotient (SQ) is a way of quantitatively representing the Phase I shearing edges on a molar tooth. Ordinary or phylogenetic least squares regression is fit to data on log molar length (independent variable) and log sum of measured shearing crests (dependent variable). The derived linear equation is used to generate an 'expected' shearing crest length from molar length of included individuals or taxa. Following conversion of all variables to real space, the expected value is subtracted from the observed value for each individual or taxon. The result is then divided by the expected value and multiplied by 100 . SQs have long been the metric of choice for assessing dietary adaptations in fossil primates. Not all studies using SQ have used the same tooth position or crests, nor have all computed regression equations using the same approach.
\end{abstract}

Understanding how the skeletons of living species have been molded by environmental selective pressures is an important goal for many evolutionary biologists and paleoanthropologists. Much research has been focused on elucidating the functional and evolutionary mechanisms by which the great diversity of dental morphologies has arisen. Any adaptive explanation for a correlation between a morphological variable and a behavioral attribute must identify a linking functional (biomechanical) "cause" (Kay and Cartmill, 1977). Research by Kay and others (Hiiemae and Kay, 1973; Kay and Hiiemae, 1974; Kay, 1975, 1978; Kay et al., 1978; Anthony and Kay, 1993) has articulated a detailed biomechanical framework for the functional significance of morphological variation in teeth based on aspects of tooth form and wear patterns (Kay and Hiiemae, 1974), which has also been supported by in vivo jaw and tooth movements of living taxa, and by an established relationship between crest length and the size of chewed food particles (Sheine and Kay 1977, 1982). These investigations culminated in the establishment of a metric called a shearing quotient (SQ), which is a measure of whether an animal has a longer or shorter sum of Phase I shearing crest lengths in proportion to its molar length relative to other taxa. Most studies have found that primates including larger amounts of structural carbohydrate in their diets (chitin in insects or plant fiber in leaves, buds, flowers, and stems) have relatively longer shearing crests for their tooth length than frugivores and hard-object feeders (e.g., Kay, 1975, 1978; Kay et al., 1978; Anthony and Kay, 1993; Strait, 1993a,b; Kirk and Simons, 2001; Strait, 2001; Kay, 2004; Bunn et al., 2011). A recent exception to this general finding was reported by Winchester et al. (2014) who found instead no significant
Here we focus on re-analyzing the data of one recent study to investigate the magnitude of effects of variation in 1) shearing crest inclusion, and 2) details of the regression setup. We assess the significance of these effects by the degree to which they improve or degrade the association between computed SQs and diet categories. Though altering regression parameters for SQ calculation has a visible effect on plots, numerous iterations of statistical analyses vary surprisingly little in the success of the resulting variables for assigning taxa to dietary preference. This is promising for the comparability of patterns (if not casewise values) in SQ between studies. We suggest that differences in apparent dietary fidelity of recent studies are attributable principally to tooth position examined. Am J Phys Anthropol 000:000-000, 2014.

2014 Wiley Periodicals, Inc.

difference between folivores and frugivores in their analyses. In this technical report, we assess the dataset of Winchester et al. (2014) in greater detail in order to evaluate whether the lack of a frugivore/folivore signal in their data is explained by certain methodological differences between previous applications of $\mathrm{SQ}$ analysis and that executed by Winchester et al. (2014). By isolating the methodological factors that lead to different perspectives on functional differentiation between folivores and frugivores, we expect to arrive at a more precise understanding of the of form-function links in the primate dentition.

In particular, Winchester et al.'s (2014) approach differed in various respects from previous applications of

\footnotetext{
Additional Supporting Information may be found in the online version of this article.

Grant sponsor: NSF; Grant numbers: DDIG BCS 0622544 (DMB), BCS 1304045 (DMB \& EMS), DDIG BCS 1341120 (JMW), BNS 0851272 (RFK); Grant sponsor: Leakey Foundation (DMB \& EMS); Grant sponsors: Evolving Earth Foundation (DMB), American Society of Mammalogists (DMB), and AAPA Professional Development Grant (DMB).
}

*Correspondence to: Doug M. Boyer, Department of Evolutionary
Anthropology, Duke University, Box 90383, Durham, NC 27708. E-mail: doug.boyer@duke.edu

Received 4 April 2014; accepted 8 September 2014

DOI: 10.1002/ajpa.22619

Published online 00 Month 2014 in Wiley Online Library

(wileyonlinelibrary.com). 
SQ (e.g., Kay et al., 1978). Winchester et al. (2014) built on another study whose methods it followed (Bunn et al., 2011) and with which much of the non-platyrrhine sample is overlapping. In fact, the main distinction between the two studies is the addition of platyrrhine data to the more recent one. Winchester et al.'s (2014) goal was to assess the degree to which ecologically salient patterns in tooth morphology extend between "prosimians" and platyrrhines. Bunn et al. (2011) found SQ patterns among "prosimians" to match previous work for the most part; thus, no immediate questions about comparability to earlier SQ studies were raised. The unexpected lack of differentiation between folivores and frugivores in Winchester et al.'s (2014) data has motivated the closer look we now take.

Some of the differences between Winchester et al. (2014) and previous studies that may affect whether ecological dietary groups can be discerned include which shearing crests were measured (Fig. 1), how regression equations for SQ were calculated, the formulae used to compute $\mathrm{SQ}$, and the taxonomic composition of ecological groups examined. These variables are directly addressed in the present study.

There are additional differences that we cannot directly address at this time, but which should eventually be evaluated: 1) While shearing crest lengths have been traditionally measured under a microscope fitted with a reticle, Bunn et al. (2011) and Winchester et al. (2014) were the first to use digital 3D surface models of teeth. Thus, their crest length measurements reflect the contour of the tooth surface more closely than most previous datasets (Fig. 1): a likely exception is the dataset of Strait (1993, 2001). She used a reflex microscope that allowed contour-following measurements on shearing crests of physical specimens. 2) Winchester et al. (2014) were the first to utilize the second lower molar (m2) in computing SQ for platyrrhines. Other studies conventionally use the $\mathrm{m} 1$. Winchester et al.'s (2014) basis for this break from tradition was the desire to compare homologous structures between platyrrhines and "prosimians": traditional SQ studies use the $\mathrm{m} 2$ for non-platyrrhines.

\section{METHODS}

In brief, $\mathrm{SQ}$ is measured as follows (see below for more detail): a least squares regression is fit to data on natural log molar length as independent variable and the natural $\log$ sum of measured shearing crests (of the same molar tooth) as dependent variable. The set of specific shearing crests measured has differed in various studies, as has choice of specific molar (Anthony and Kay, 1993; Strait, 1993; Kirk and Simons, 2001, Bunn et al., 2011; also see below). In addition, in some analyses a subset of all measured data is used to calculate the least squares regression, due to possible issues of allometry (Anthony and Kay, 1993; Strait, 1993; also see below). The derived linear equation is used to generate an empirical "expected" shearing crest length from molar length of each included taxon. This expected value is then transformed back into arithmetic space and subtracted from the arithmetic version of the observed value for each taxon. The result is then divided by the expected value, and multiplied by 100 . The resulting value is the SQ.

Previously, the dietary signal in SQ has been evaluated by running discriminant function analyses (DFA) on data points representing individuals grouped by diet preference (from the literature of behavioral ecology) and reporting the classification success, or by using phylogenetic ANOVA and post hoc comparisons of dietary groups. In this study, we follow the exact analytical protocols for DFA and phylogenetic ANOVA as described in Winchester et al. (2014) so that our results are directly comparable to theirs. This includes the dietary coding given to taxa and also the phylogenetic tree and branch lengths used to relate included taxa. We also run additional phylogenetic ANOVAs in which platyrrhines and "prosimians" are separated. This was not done by Winchester et al. (2014) and therefore, the fidelity of the signal within each group was not previously investigated.

\section{Methodological details omitted from Winchester et al. (2014)}

Because we re-analyze measurements used in SQ calculation by Winchester et al. (2014), it is necessary to revisit their methods. Most details on how they took their measurements, what crests they included, and which teeth and taxa they used can be found in their paper or in Bunn et al. (2011). It is however, necessary to explain select details of analytical protocols used for SQ calculation and analysis by Winchester et al. (2014). These are limited to important details that were omitted from the original article (DMB and JW have personal knowledge of those details), but which help establish the degree to which their results should be comparable to those obtained in other studies. Methodological details omitted from Winchester et al. (2014) include the following:

a. Winchester et al. (2014) state on page 32, lines 25-30, that "To calculate both [SR and SQ], shearing crest lengths were measured from each surface using the point distance tool of Geomagic Studio 10 (Geomagic). For further details and repeatability tests for this method of measuring shearing crests, see Bunn et al. (2011)." This statement implies that their crest measurement protocol followed Bunn et al. (2011), which is inaccurate because it was revised with respect to the "protocristid" crest (see Bunn et al., 2011, their figure 1: they refer to the segment bounded by Landmarks 2 and 3), such that the method of Winchester et al. (2014) differs in only representing the length of the buccal half of the protocristid instead of the entire protocristid as in Bunn et al. (2011). The method of Winchester et al. (2014) is more in-line with protocols used by Anthony and Kay (1993) and Kirk and Simons (2001). Figure 1 shows different measurement protocols of various studies discussed here.

b. Winchester et al. (2014) did not provide the regression coefficients used for SQ calculation in their study. These are now provided below as reg\#1 for platyrrhine and prosimian samples (Table 1). Winchester et al. (2014) used separate equations for platyrrhines and prosimians. Therefore, whenever results of combined analyses including SQ of platyrrhines and prosimians are presented, the analyses used numbers based on two different regression equations.

c. On page 32 , line 34 of Winchester et al. (2014) the authors state that "SQ per specimen was calculated by the method of Anthony and Kay (1993)" but there are significant differences between the two methods for SQ calculation as follows:

i. Anthony and Kay (1993) did not include the postentocristid crest in their total shearing crest length measurement, whereas Winchester et al. 

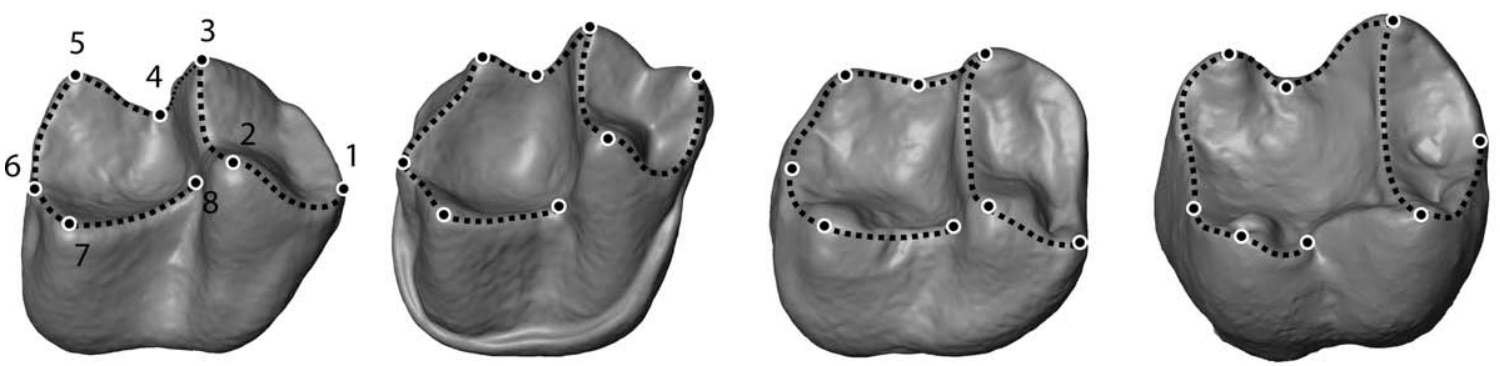

A. Landmarks and crests as measured in Bunn et al. (2011)
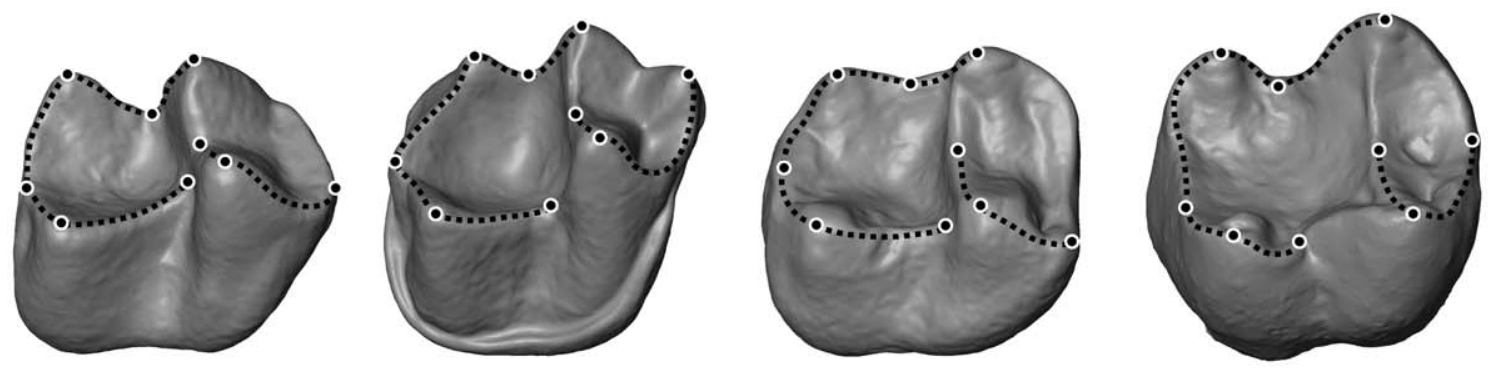

B. Landmarks and crests as measured in Winchester et al. (2014)
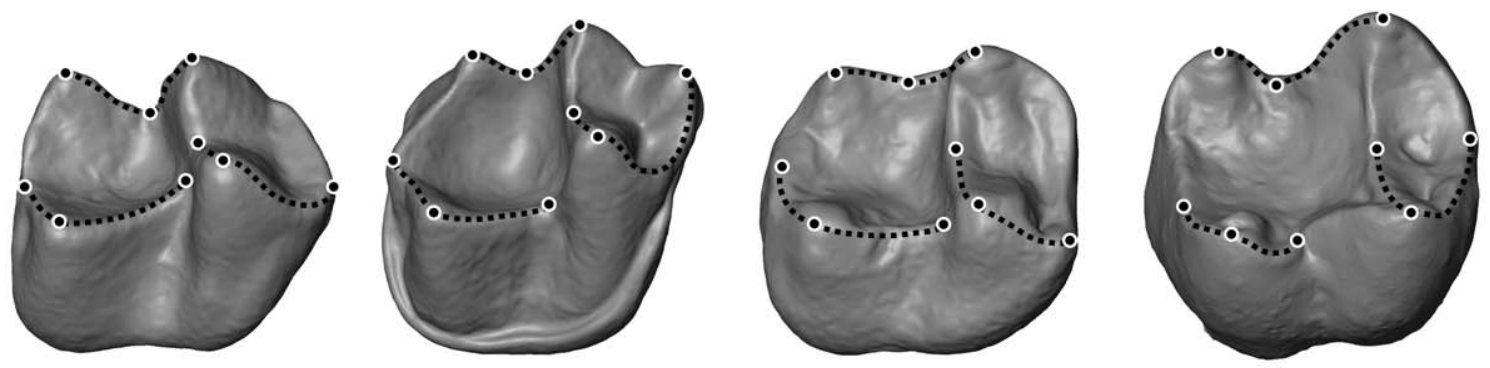

C. Landmarks and crests as included for analyses of this study lacking the post-entocristid crest
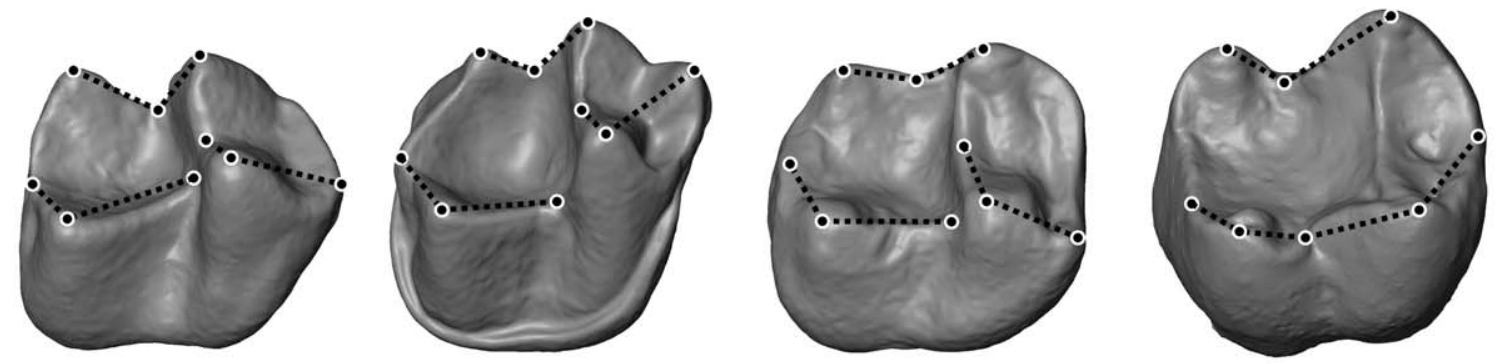

D. Approximate landmarks and crests as traditionally measured and included (e.g., in Anthony \& Kay 1993) with more typically available equipment, such as microscope with reticle.

Fig. 1. Illustration of measurement protocols from different studies discussed here. The numbered landmarks represent the end points of crest-length measurements. Labels for these numbered points are given in Bunn et al. (2011: their figure 1). See Figure 2 for more extensive labeling of tooth anatomy discussed in the main text. Parts A-C represent modifications on an approach and dataset originally analyzed by Bunn et al. (2011). Crest measurements in these datasets were taken on 3D models of teeth and follow the contour of the crest relatively closely. In practice, two to five straight-line segments are used to trace each crest. In $\mathbf{A}$, the measurement protocol of Bunn et al. (2011) is shown. It contrasts with B, that of Winchester et al. (2014), in the representation of the protocristid segment (between points 2 and 3): Winchester et al. (2014) changed their measurement of this region by including only the buccal half of the crest. Overall, the protocol shown in B matches very closely that used by Strait (2001) for example. C, shows the modification on crest inclusion where the segment between 5 and 6 [the postentocristid, or "crest $6 \mathrm{~b}$ " by Strait (2001)] is excluded (this measurement set is used in AK93-2, V2, V4, V6, V8, V10, and V12: see Tables 1-4). D shows how crests would be measured using more traditional equipment (microscope with reticle). Researchers wishing to produce datasets more directly comparable with, for example, Kay (1975), Kay et al. (1978), and Anthony and Kay (1993) should emulate the protocol shown in D in their measurements. Note the difference between the positions of the crest functionally equivalent to the protocristid on Lagothrix in $\mathbf{D}$ compared to the others. Detailed examination of wear-facets justifies the protocol in $\mathbf{D}$ as shown in Figure 2. Clearly, these different measurement methods will result in different values for total crest length when used on the same specimens. Scale bars (shown beneath the specimen numbers) $=2 \mathrm{~mm}$. 
(2014) did so. The major justification for Anthony and Kay's (1993) approach is that the postentocristid does not usually develop a Phase I shearing crest in platyrrhines (or other athropoids). This is because the postentocristid is associated with hypocone occlusion and hypocones are variably present in platyrrhines. Due to lack of a hypocone in Tarsius, it would seem omission of the postentocristid crest is justified in it as well; however, other authors besides Bunn et al. (2011) and Winchester et al. (2014) have included it when measuring "prosimians" (Strait, 1991, 1993a,b, 2001).

ii. Anthony and Kay (1993) computed their regression for SQ using 11 frugivorous (including hardobject feeding) platyrrhine taxa, but excluding more folivorous Brachyteles and Alouatta species, whereas Winchester et al. (2014) included only species in four genera categorized as "frugivorous" by them, including Aotus, Ateles, Callicebus, and Lagothrix. Anthony and Kay (1993) reasoned that their approach was appropriate specifically because the folivores of the platyrrhine radiation have disproportionately large teeth for their body size, and confound the use of molar length as a proxy for body size when computing a regression. Thus, for Anthony and Kay (1993), the criterion for choosing taxa for the SQ regression was not "they must be frugivores" it was "they must not be committed folivores." This leads to another point regarding Winchester et al.'s (2014) methods:

iii. Winchester et al. (2014) used only strepsirrhine frugivores for the "prosimian" regression. However, because the tooth size range of folivores is not so extremely shifted relative to that of other strepsirrhine diet groups (e.g., Varecia is a frugivore that has teeth close in size to Propithecus and Indri which are folivores, and there are various smallbodied and small-toothed folivores - like Avahi and Lepilemur), Kirk and Simons (2001) noted that no omissions are necessary when generating regressions for computation of $\mathrm{SQs}$ in strepsirrhines. On the other hand, Strait (1993) observed body size skew in her sample of "prosimians" and chose to use ratios instead of residuals. Taking all this into account, it was possibly incorrect of Winchester et al. (2014) to use a "frugivore only" protocol for either platyrrhines or prosimians.

iv. Anthony and Kay (1993) used species mean data to compute their regression for $\mathrm{SQ}$ calculation, whereas Winchester et al. (2014) used individual data points.

v. After computing "expected" natural log shearing crest lengths from their regression using natural log m1 length, Anthony and Kay (1993) convert regression predictions and observed values back into arithematic units before computing a quotient. Although Anthony and Kay (1993) do not state this themselves, their approach can be verified by using data they provide to compute SQs and by then comparing those values to the SQs they report. Although log-space residuals can be used without dividing them by an expected value as they shall be uncorrelated with it, dividing logspace residuals by their expected value will introduce a correlation between magnitude of variation and the expected shearing crest value (as well as molar length). In other words, a size effect will be introduced. Neither Bunn et al. (2011) nor Winchester et al. (2014) antilog-transformed their data before computing SQ, meaning their datasets probably suffer from an erroneous size-effect and are not ideal for assessing functional variation over a wide range of tooth sizes.

\section{Noted errors in Winchester et al. (2014) ${ }^{1}$}

a. We discovered an extreme outlier in SQ values among the data of Winchester et al. (2014). One specimen of Cebus apella (USNM 461384) exhibited the maximum SQ of any platyrrhine in their dataset by a wide margin, whereas other individuals of Cebus had relatively low SQs (see Winchester et al., 2014, Supporting Information Table S3). Re-measurement of this specimen returned much more average values verifying that there was an error in recording the original numbers.

b. On page 37, Table 6, in the column titled "SQ", three post hoc comparisons are bolded as significant. In the "Sig" row of that column, the number " 3 " indicates that only three comparisons were significant. However, data in the "Ins. vs. Ho." row of the "SQ" column shows $P=0.004$, meaning that comparison was also significant by the chosen critical value. Thus, actually four post hoc comparisons out of 10 were significant for SQ in Winchester et al.'s (2014) original analysis, though this did not include the comparison between folivores and frugivores, which is correctly reported by them as nonsignificant.

\section{Analyses}

We modified the approach used by Winchester et al. (2014) to make it more comparable to previous studies. To make our study more repeatable and more easily evaluated, we also provide a Supporting Information table that includes all measured variables for every specimen (Supporting Information Table S1) to accompany Winchester et al.'s (2014) Supporting Information Table S3 (which contains only computed variables). In addition, we provide a table that gives species mean values used in phylogenetic comparative analyses (Supporting Information Table S2). We provide the nexus file representing the phylogenetic tree used in phylogenetic comparative analyses (Supporting Information Doc 1). This tree is the same as that used in Winchester et al. (2014) and was downloaded from version 3.0 of the official $10 \mathrm{k}$ trees site (Arnold et al., 2010). Finally, we provide Supporting Information Table S3, which gives SQ by individual for 14 different iterations of computation evaluated in this study.

Before re-analyzing the dataset, we removed USNM 461384, re-computed diet-group means and standard errors, and re-ran DFA (Table 2, V1**) and phylogenetic ANOVAs (Table 3: V1**) using the methods and programs described in Winchester et al. (2014). Note that classification rates improved from $57.7 \%$ (as reported by Winchester et al., 2014) to $60 \%$ (Table 2 : V1**) as a result. On the other hand, the pattern of significance for phylogenetic ANOVA did not change (Table 3 ).

\footnotetext{
${ }^{1}$ It was brought to our attention that on page 30 , column 2 , line 1 , of Winchester et al. (2014) the following citations should have been included after the word "diet": (Cuozzo and Sauther, 2006; Millette et al., 2009; Sauther and Cuozzo, 2009; Campbell et al., 2012; Cuozzo and Sauther, 2012).
} 


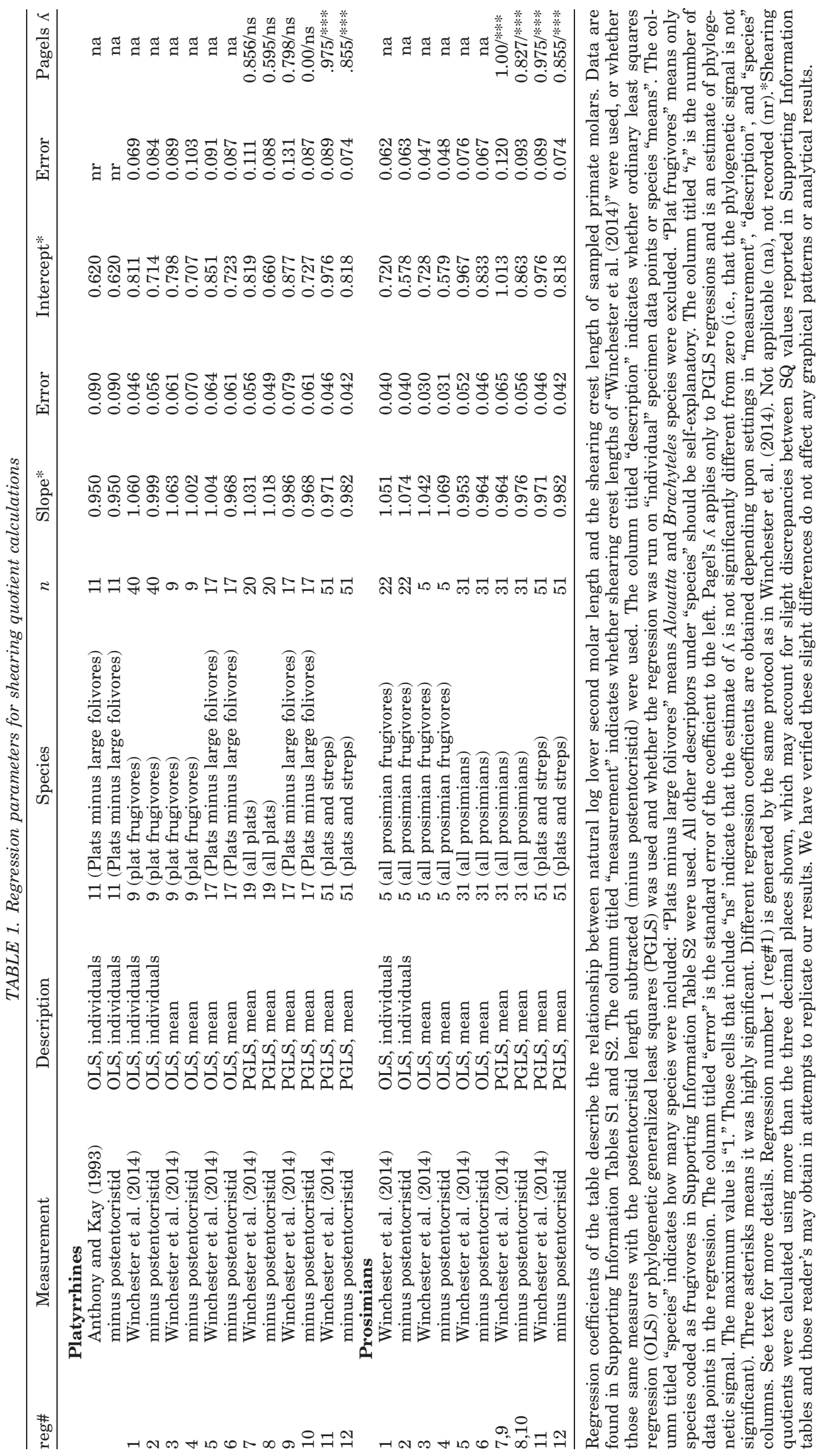




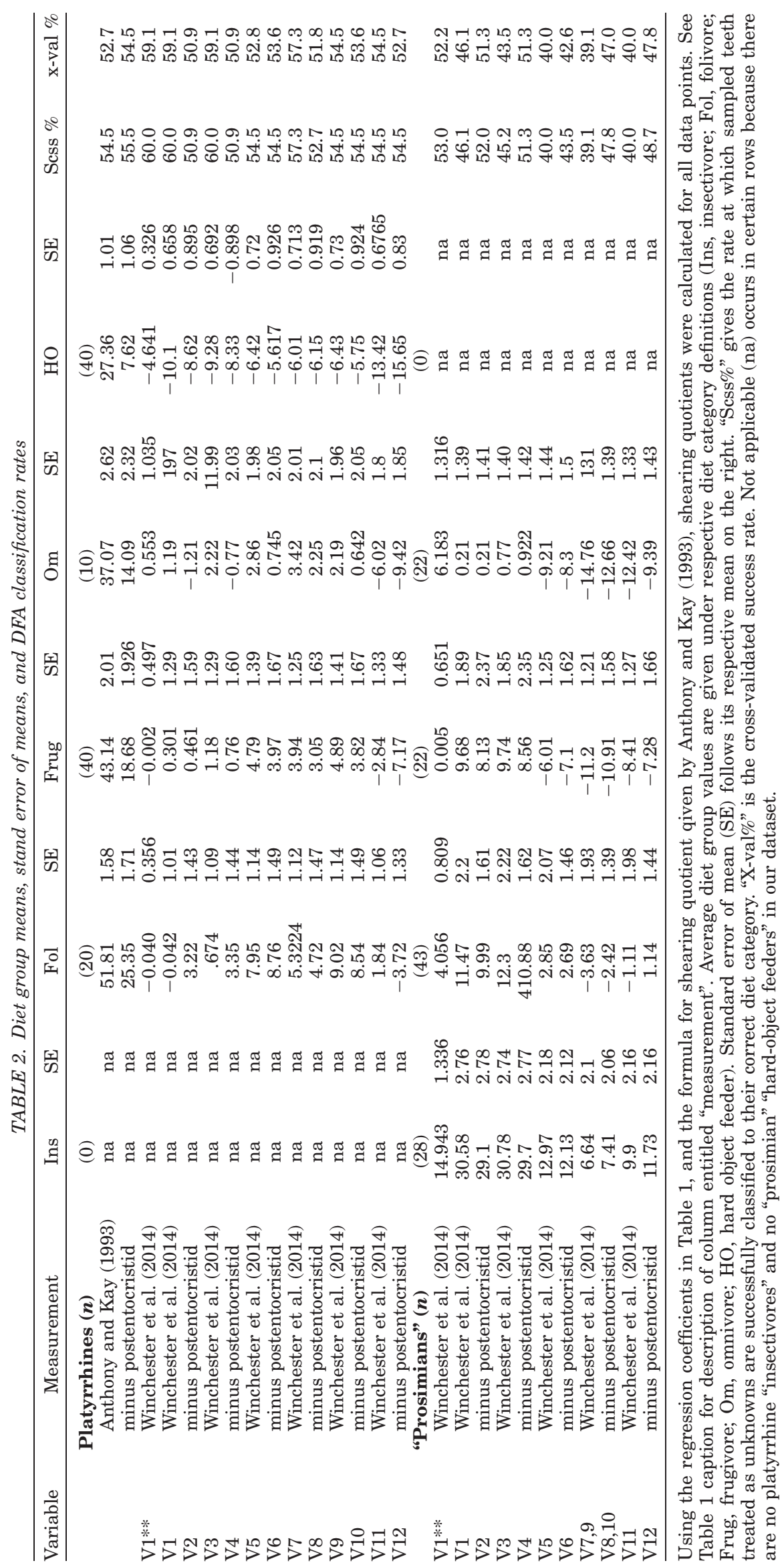


RECENT USE OF SHEARING QUOTIENTS

TABLE 3. Post hoc comparisons for phylogenetic ANOVA on different versions of $S Q$

\begin{tabular}{|c|c|c|c|c|c|c|c|c|c|c|c|c|}
\hline & $\lambda$ & $\mathrm{IvF}$ & $\mathrm{IvO}$ & IvFr & $\mathrm{IvH}$ & $\mathrm{FvO}$ & FvFr & $\mathrm{FvH}$ & $\mathrm{OvFr}$ & $\mathrm{OvH}$ & FrvH & sig \\
\hline $\mathrm{V} 1 * *$ & 0.987 & 0.0027 & 0.0044 & 0.0020 & 0.0015 & $\mathrm{~ns}$ & $\mathrm{~ns}$ & $\mathrm{~ns}$ & $\mathrm{~ns}$ & $\mathrm{~ns}$ & $\mathrm{~ns}$ & $4 / 4$ \\
\hline V1 & 0.973 & 0.0117 & 0.0043 & 0.0059 & 0.0022 & $\mathrm{~ns}$ & $\mathrm{~ns}$ & $\mathrm{~ns}$ & ns & ns & $\mathrm{ns}$ & $2 / 4$ \\
\hline V2 & 0.841 & 0.0434 & 0.0027 & 0.0016 & 0.0006 & $\mathrm{~ns}$ & $\mathrm{~ns}$ & 0.0486 & $\mathrm{~ns}$ & $\mathrm{~ns}$ & $\mathrm{~ns}$ & $3 / 5$ \\
\hline V3 & 0.970 & 0.0135 & 0.0042 & 0.0065 & 0.0021 & $\mathrm{~ns}$ & $\mathrm{~ns}$ & $\mathrm{~ns}$ & $\mathrm{~ns}$ & ns & $\mathrm{ns}$ & $2 / 4$ \\
\hline V4 & 0.838 & 0.0450 & 0.0026 & 0.0016 & 0.0006 & $\mathrm{~ns}$ & $\mathrm{~ns}$ & 0.0459 & $\mathrm{~ns}$ & $\mathrm{~ns}$ & $\mathrm{~ns}$ & $3 / 5$ \\
\hline V5 & 0.937 & $\mathrm{~ns}$ & 0.0072 & 0.0442 & 0.0063 & $\mathrm{~ns}$ & ns & 0.0508 & ns & ns & $\mathrm{ns}$ & $0 / 3$ \\
\hline V6 & 0.678 & $\mathrm{~ns}$ & 0.0019 & 0.0166 & 0.0015 & 0.0128 & 0.0207 & 0.0033 & $\mathrm{~ns}$ & $\mathrm{~ns}$ & $\mathrm{~ns}$ & $3 / 6$ \\
\hline V7 & 0.926 & $\mathrm{~ns}$ & 0.0066 & 0.0355 & 0.0040 & $\mathrm{~ns}$ & $\mathrm{~ns}$ & 0.0527 & $\mathrm{~ns}$ & ns & $\mathrm{ns}$ & $1 / 3$ \\
\hline V8 & 0.681 & $\mathrm{~ns}$ & 0.0013 & 0.0113 & 0.0009 & 0.0343 & 0.0679 & 0.0069 & $\mathrm{~ns}$ & $\mathrm{~ns}$ & $\mathrm{~ns}$ & $2 / 6$ \\
\hline V9 & 0.933 & $\mathrm{~ns}$ & 0.0096 & 0.0518 & 0.0072 & $\mathrm{~ns}$ & $\mathrm{~ns}$ & 0.0444 & ns & ns & $\mathrm{ns}$ & $0 / 3$ \\
\hline V10 & 0.704 & $\mathrm{~ns}$ & 0.0026 & 0.0208 & 0.0020 & 0.0193 & 0.0300 & 0.0046 & $\mathrm{~ns}$ & ns & $\mathrm{ns}$ & $3 / 6$ \\
\hline V11 & 0.951 & $\mathrm{~ns}$ & 0.0082 & 0.0388 & 0.0079 & $\mathrm{~ns}$ & $\mathrm{~ns}$ & 0.0608 & $\mathrm{~ns}$ & ns & $\mathrm{ns}$ & $0 / 3$ \\
\hline V12 & 0.747 & $\mathrm{~ns}$ & 0.0017 & 0.0064 & 0.0009 & 0.0513 & 0.0374 & 0.0075 & $\mathrm{~ns}$ & $\mathrm{~ns}$ & $\mathrm{~ns}$ & $2 / 5$ \\
\hline
\end{tabular}

Bonferroni correction to $\alpha=0.05$ is $\alpha=0.005$ for 10 comparisons. "sig" column gives number of comparisons significant at $P<0.005 / P<0.05$. V1** is original SQ data from Winchester et al. (2014), with the exception that one data point of Cebus apella, which was an outlier, has been removed (see text). Note it shows the same pattern of significance at $P<0.005$ as in Winchester et al. (2014). These comparisons were done on an "all primates" sample of 51 species, both platyrrhines and "prosimians" (Supporting Information Table S2). As discussed in the text, shearing quotients for each dataset were generally generated from separate regressions for platyrrhines and strepsirrhines with the exception of V11-12. Note the major difference between V1** and V1 is that SQ was calculated from logged data in the first, while in V1-12, data were back-transformed to arithmetic values before SQ was computed. $K=$ Pagel's $\Lambda$.

Crest inclusion. To assess the effect of including or excluding the postentocristid segment length on SQ patterns, we created a version of Winchester et al.'s (2014) shearing crest dataset without the postentocristid segment length (Winchester et al. [2014] retained a copy of their dataset in which all individual crest values were separated for each specimen). The effect on SQ regression parameters of not including the postentocristid in the shearing crest length is shown in Table 1 (reg\#2). The effect on diet group means, and DFA success is shown in Table 2 (V2). The effect on phylogenetic ANOVA is shown in Table 3 (V2).

Regression construction. When we arrive at points "ii-iv", which have to do with constructing a regression analysis for SQ computation, we encounter some difficulty in revising the analyses of Winchester et al. (2014) to make them resemble those of Anthony and Kay (1993) because Winchester et al. (2014) did not have access to all of the same taxa. Specifically, the sample at hand lacks tamarins and marmosets. Therefore, we approached evaluating the significance of the discrepancy between Winchester et al.'s (2014) SQ regression protocols and those of Anthony and Kay (1993) in three ways. First, we assessed the effect of using species mean data for the regression with the original four genera, representing nine species from Winchester et al. (2014). We evaluated this for Winchester et al.'s (2014) original shearing crest dataset (again excluding the Cebus outlier) and the new shearing crest dataset for which the postentocristid had been removed (Tables 1 and 2: platyrrhine reg\#s 3-4; Table 3: V3-V4). Similarly, for the prosimian sample we used species mean data from five strepsirrhine frugivore species (Tables 1 and 2: prosimian reg\#s 3-4; Table 3: V3-V4). Next we used species mean data for all platyrrhine taxa except the two species of Alouatta and one species of Brachyteles (Tables 1 and 2: platyrrhine reg\#s 5-6; Table 3: V5-V6). This resulted in a regression with 17 taxa for platyrrhines. For "prosimians," following Kirk and Simons (2001) we used species mean data of all taxa in Winchester et al.'s (2014) sample $(n=31)$. Again, each of these analyses was run on both the original dataset from Winchester et al. (2014) and that excluding the postentocristid segment (Tables 1 and 2: prosimian reg\#s 5-6; Table 3: V5-V6). The V6 treatment comes closest to the analytic protocol described by Anthony and Kay (1993) and Kirk and Simons (2001) for platyrrhines and "prosimians." We also tested the effect of using Anthony and Kay's (1993) regression coefficients on our crest data (first two rows of Tables 1 and 2). Finally, we computed regression equations for $\mathrm{SQ}$ using PGLS in $\mathrm{R}$ (with the Caper package as described in Winchester et al. [2014]) for three different datasets: one using all species mean platyrrhine data (platyrrhine reg \#s 7-8), another excluding Alouatta and Brachyteles (platyrrhine reg \#s 9-10), and a final one using species mean data on molar length and shearing crest length that includes all primates of the sample (reg \#s 11-12).

Note that there is a regression \#1 for platyrrhines, as well as for "prosimians" (Table 1). Each platyrrhine regression is paired with a "prosimian" regression on the basis of using similar protocols for generating both regressions. For instance, regression \#1 for platyrrhines and regression \#1 for "prosimians" are comparable in that they are both based on individual specimens instead of species means, both utilize only "frugivores" of their respective clades, both are run using OLS regression analysis, and both use the original shearing crest data of Winchester et al. (2014). When conducting comparative analyses that require combining platyrrhine and "prosimian" samples (e.g., phylogenetic ANOVA) the platyrrhine regression \#1 would be used for the platyrrhines and the "prosimian" regression \#1 would be used for the "prosimians". A combined sample of "prosimians" and platyrrhines would not generally be used to generate a more broadly applicable SQ regression due to the possibility of clade offsets in scaling relationships between the two groups (e.g., Kirk and Simons, 2001). Misleading regression results based on such phylogenetic correlation should be diminished using phylogenetic comparative methods. Therefore, one set of regressions, 11-12, is based on an "all primate" sample (Supporting Information Table S2).

Finally note that platyrrhine regressions \#7 and \#9 differ only by whether large folivores were used in the regression computation. The same difference applies to \#8 and \#10. As discussed above, we had no similar justification for 


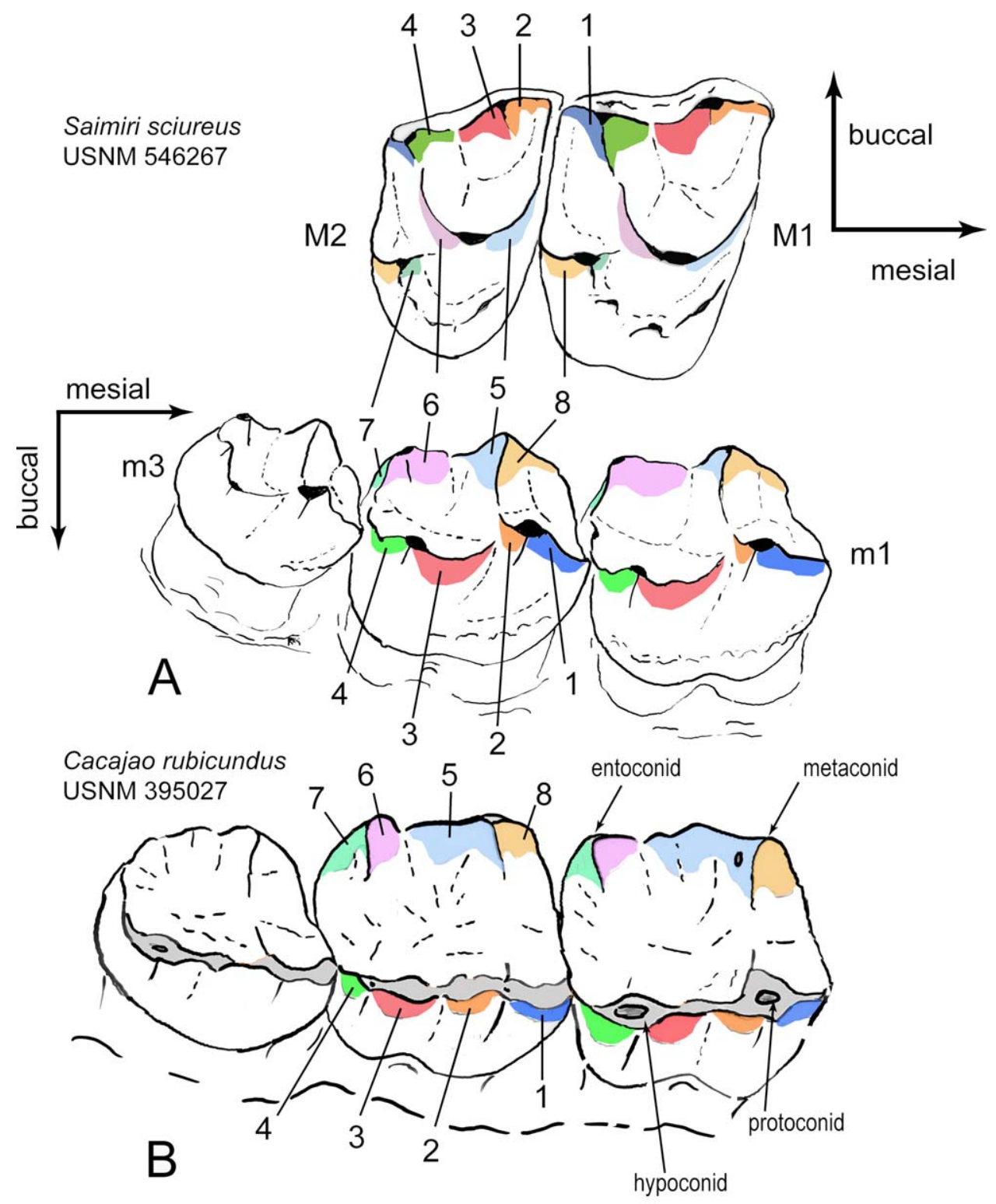

Fig. 2. Occlusal views of right maxillary second and third molars and oblique lateral views of right mandibular first through third molars of A, Saimiri sciureus (USNM 546762) and B, Cacajao rubicundus (USNM 395027) (not to same scale). The leading edges of eight functional crests are labeled, as are the wear surfaces distal to them. Anthony and Kay (1993) measured the leading edges of crest lengths 1-6. Crests 7 and 8 (associated with the margins of the hypocone and trigonid basin) were not measured (see also Fig. 1D). Numbering system of the crests follows Kay (1977). In Cacajao, as in many platyrrhines, the protocristid is rotated into a mesiodistal orientation leaving crest 1 restricted to the buccal edge of the crown, so a measurement (on the mandibular second molar) from the notch between the metaconid and the protoconid does not accurately represent the length of that crest. Illustration modified after Allen et al. (in review).

removing large folivores from the "prosimian" regression. Therefore, "prosimian" regressions \#7 and \#9 are identical, while \#8 and \#10 are also identical.

Quotient calculation. Clearly, if quotients are to be used, the more appropriate method of computing them is with antilogged data. Therefore, all SQs are based on using arithmetic versions of measured shearing crest lengths, and antilogged versions of predicted shearing crest lengths. Only the variable $\mathrm{V} 1^{* *}$ is presented with SQ computed from logged values as per the protocol of Winchester et al. (2014). This is in order to provide a comparison between results of analysis of Winchester et al.'s (2014) protocol and that used here.

Taxonomic inclusion. Examining Winchester et al.'s (2014) results (see their figure 2 and table 2) leads to the impression that a lack of differentiation among platyrrhine folivores and frugivores drives the lack of differentiation between folivores and frugivores generally in their phylogenetic ANOVA post hoc comparisons (Winchester et al., 2014: their table 6). A diet-group ANOVA is not even conducted by Bunn et al. (2014). Thus, we conduct ANOVA's by diet group here for the "prosimian" sample alone, and the 
platyrrhine sample alone. However, using a species mean approach for these reduced samples creates statistical issues. For the platyrrhine sample, only two species of Saimiri can be categorized as omnivores. Therefore they must be removed from the analyses. This means platyrrhine ANOVA's include only three groups: folivores, frugivores, and hard-object feeders. This dictates a different bonferroni corrected critical value of $0.05 / 3=0.01666$ for these analyses. Likewise, our "prosimian" analysis only includes eight comparisons among four diet groups (no hard object feeders) and has a critical value of $0.05 / 8=0.008333$.

Removing Saimiri species presents another issue: estimates of Pagel's lambda are known to be unreliable for $n<20$ (Blomberg et al., 2003). Instead of re-analyzing all 14 SQ iterations with these analyses, we do this for three of the variables: V1 (the original dataset utilizing the correct computation of SQ including antilogging estimated values prior to quotient calculation), V2 (the original dataset and regression set-up with the post entocristid removed), and V6 (the version of SQ computation that comes closest to the protocols used by Anthony and Kay (1993).

\section{RESULTS \\ Quotient calculation}

Though discussion of quotient calculation logically comes towards the end of our methods description above, it must come first here, since it applies to all iterations of analysis. To assess affects of this change we computed variable set 1 (based on regression 1 in Table 1) using logged data (V1**) and with antilogged data (V1). All subsequent variables sets are computed on antilogged data. Surprisingly there is no difference in the classification success between V1** and V1 (Table 2; Fig. 3). Even more surprisingly, the number of significant post hoc comparisons goes down from V1** to V1 (Table 3).

\section{Crest inclusion}

Changing Winchester et al.'s (2014) protocol by simply removing the postentocristid segment length from each shearing crest value actually resulted in poorer classification rates for platyrrhines (Table 2: compare V1 to V2, V3 to V4, etc.). However, correct classification rates for prosimians increased. As well, the number of significant post hoc comparisons found by phylogenetic ANOVA also increased (Table 3: compare V1 to V2, V3 to V4, etc.). However, none of the Phylogenetic ANOVA's recovered significant post hoc differences between folivores and frugivores (given the corrected critical value of $\alpha=0.005$ ), in contrast to some of the dental topographic variables evaluated in Winchester al. (2014). Variables V6, V10, and V12 resulted in differences between folivores and frugivores at $P<0.05$, which can be considered significant if alpha corrections are ignored (Table 3).

\section{Regression construction}

Regressions 3-12 (Table 1) represent alternative ways of setting up the regression to be used for $\mathrm{SQ}$ calculation. Surprisingly, there was no improvement over the original protocol of Winchester et al. (2014) for any of these trials (Tables 2 and 3). Using the regression coefficients from Anthony and Kay (1993) also did not notably improve the dietary correlation of the data.

\section{Taxonomic inclusion}

Separating data into platyrrhine and "prosimian" groups before running phylogenetic ANOVA shows no significant distinction between folivores and frugivores in any ANOVA for any variable assessed (V1, V2, or V6; see Table 4). However, the probability of similarity between folivores and frugivores decreases going from V1 to V2, and from V2 to V6. Pagel's $\Lambda$ is zero for the platyrrhine analyses. Whether this is accurate or not, it means the results are equivalent to standard ANOVA. The "prosimian" ANOVA returned high values of Pagel's $\Lambda$, meaning that its results may differ from standard ANOVA. Results of standard ANOVA, followed by pairwise $t$-tests serving as post hoc comparisons returned a greater number of significantly different groups for V1-V2 (3 instead of 2), but the difference between folivore and frugivore groups still did not reach significance with the bonferroni correction (Table 4). On the other hand, use of a more liberal, Dunn-Sidak correction leads to a significant result for the "prosimian" folivore-frugivore differences in V2 and V6.

\section{DISCUSSION}

Utilizing SQ computed from logged values (Tables 2 and 3: V1**) yielded a more successful classifier than when SQ was computed from antilogged values (Tables 2 and 3: V1). Even so, and despite the fact that the former approach was used in Bunn et al. (2011) and Winchester et al. (2014), we do not advocate it. It is not mathematically or statistically justifiable and the difference in success rates must be attributed to random effects, rather than a more appropriate representation of the biological system under investigation. Going forward in this paper, we use SQ computed from antilogged values, though we note there is no reason that log-space residuals could not be used on their own in place of a SQ.

Patterns in SQ data were found to be relatively robust to variations in crest inclusion and regression parameters for the dataset of Winchester et al. (2014), because none of the variations relating to these parameters in our analyses established a significant difference between folivores and frugivores (Table 3). Interestingly, our DFA results could be argued to justify the inclusion of the postentocristid in platyrrhine datasets, as well as its exclusion from "prosimian" datasets, since its inclusion consistently degraded the classification results for the former group, but improved them for the latter. We do not, however, recommend this given that it goes against more detailed understandings of the wear facet development in these groups (Kay, 1977) (Fig. 2). The lack of a strong dietary effect from crest inclusion is reassuring since other analyses of "prosimians" by (Strait, 1991, 1993a,b, 2001) have included the postentocristid when measuring shearing crests. As well, studies by King et al. (2005) and Lanyon and Sanson (1986) have taken very different approaches to shearing crest measurement. Our results suggest that these different studies have contributed ecologically consistent patterns of variance despite different choices of crest measurement. It seems reasonable to conclude that as long as the approach is consistent within a study, there is little concern for a particular degradation of dietary signal. Clearly the fewer crests measured, the more likely that signal-to-noise ratio in the data will diminish (e.g., Kay et al., 1978). However, all the methods evaluated here utilize the majority of functional crests.

With regard to taxonomic inclusion and whether or not it is better to use species means or individuals for SQ regression computation, a similar perspective can be applied: as long as the method is consistent within a study, no major statistically significant effects on the 

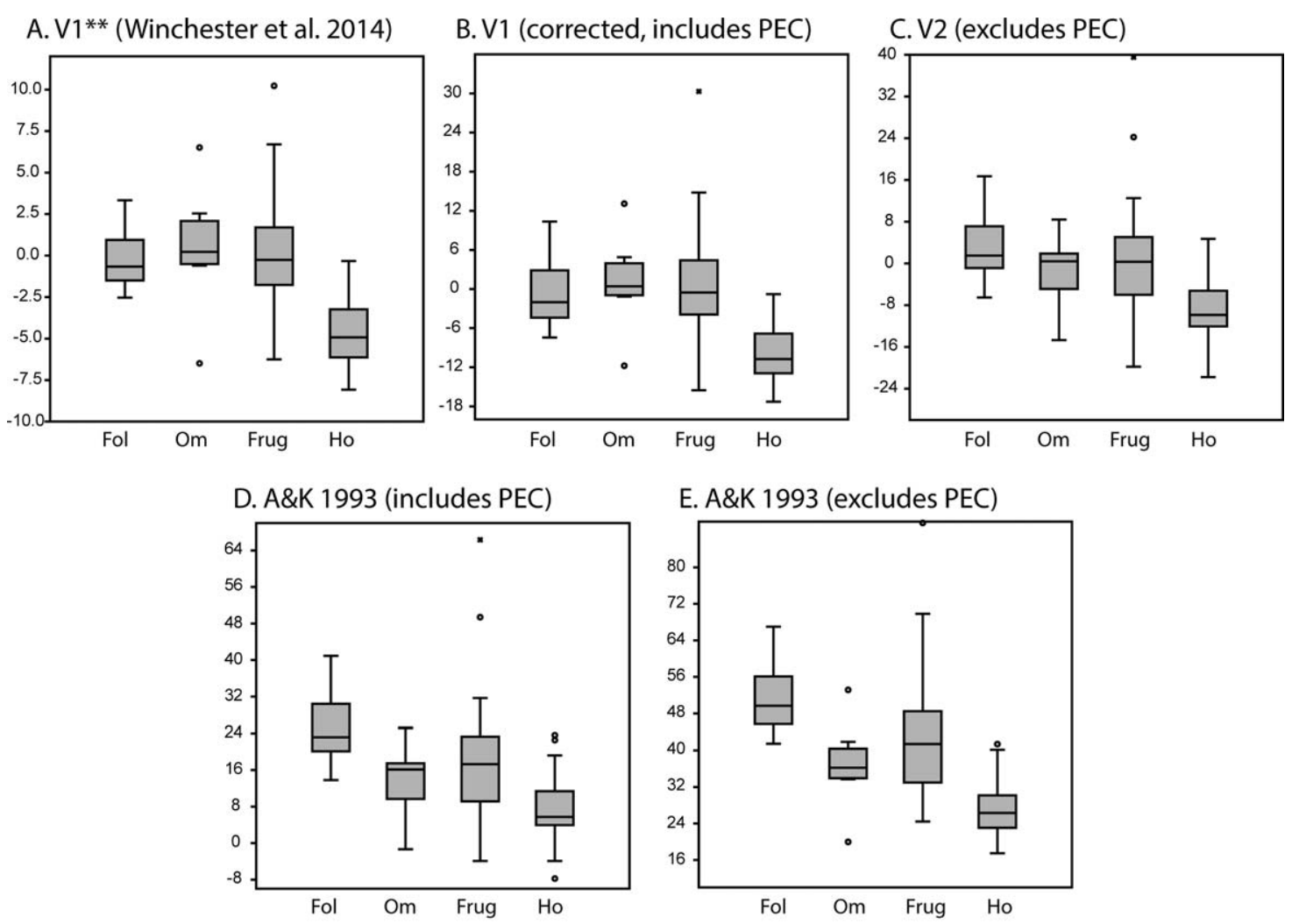

\section{F. V5 (includes PEC)}

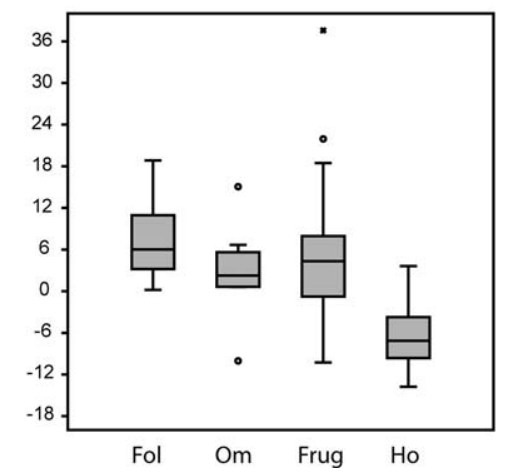

G. V6 (excludes PEC) - best

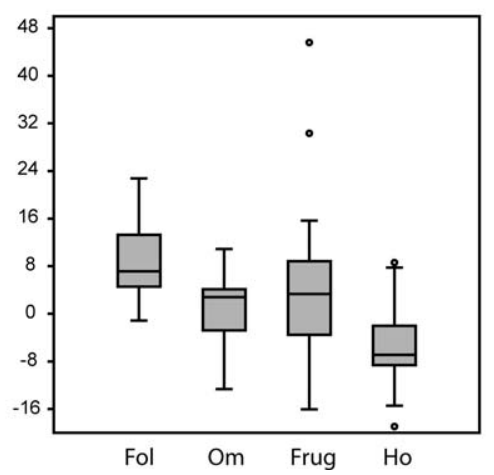

Fig. 3. Box and Whisker plots of SQ values for all platyrrhine individuals, grouped by diet category obtained using different regressions and different sets of crests. Boxes represent 25-75\% quartiles. Horizontal line represents medians. Whiskers extend at most to 1.5 times the interquartile range beyond the boxes. Points more distant are shown as outliers. Raw data and dietary categories per specimen are given in Supporting Information Tables S1 and S3. V1** plots SQs derived from original measurement, regression, and SQ computation protocol used by Winchester et al. (2014) (A). We explain issues with each of these protocols in the text. The V1 plot $(\mathbf{B})$ is based on the original measurement and regression protocol with a more correct SQ computation protocol. V2 is the original regression protocol with a different measurement protocol (excluding the postentocristid segment length) and the correct SQ computation protocol (C). "A\&K 1993" uses the regression parameters of Anthony and Kay (1993) to compute SQs based on the original measurement dataset of Winchester et al. (2014) (D) or using that dataset with the postentocristid segment removed (E). Finally, $\mathbf{F}$ and $\mathbf{G}$ show the crest lengths from Winchester et al. (2014), and those crest lengths with the postentocristid crest substracted, converted to SQs using regressions \#5 and \#6 (Table 1), respectively. The V6 iteration of crest inclusion and regression computation obtained the largest differences between folivores and frugivores, though these differences still did not reach significance in our analyses.

diet signal in the resulting SQ data will be evident. We say this because changing the regression parameters to make them more comparable to those in Anthony and Kay (1993) or changing them in other ways (using PGLS) did not improve overall classification rates (Table 2 ) or the number of significant post hoc comparisons in phylogenetic ANOVA (Table 3). On the other hand, graphical examination of the data on individuals grouped by diet suggests that regression protocols better approximating those of Anthony and Kay (1993) recovered greater distinction between platyrrhine folivores and frugivores (Fig. 3F-G). Furthermore, in phylogenetic ANOVA of V6 SQ values (the crest + regression protocol most closely approximating Anthony and Kay, 
RECENT USE OF SHEARING QUOTIENTS

TABLE 4. Post hoc comparisons for phylogenetic ANOVA on select versions of SQ for platyrrhines and prosimians treated separately

\begin{tabular}{|c|c|c|c|c|c|c|c|c|c|c|c|c|}
\hline & $\Lambda$ & $\mathrm{IvF}$ & $\mathrm{IvO}$ & $\mathrm{IvFr}$ & $\mathrm{IvH}$ & $\mathrm{FvO}$ & FvFr & $\mathrm{FvH}$ & $\mathrm{OvFr}$ & $\mathrm{OvH}$ & FrvH & sig \\
\hline \multicolumn{13}{|c|}{ Platyrrhines } \\
\hline V1 & 0.00 & NA & NA & NA & NA & NA & 0.68 & 0.0006 & NA & NA & 0.0001 & $2 / 2$ \\
\hline $\mathrm{V} 2$ & 0.00 & NA & NA & NA & NA & NA & 0.26 & 0.0009 & NA & NA & 0.001 & $2 / 2$ \\
\hline V6 & 0.00 & NA & NA & NA & NA & NA & 0.085 & 0.0003 & NA & NA & 0.001 & $2 / 2$ \\
\hline \multicolumn{13}{|c|}{ Prosimians } \\
\hline V1 & 1.0 & 0.13 & 0.005 & 0.006 & NA & 0.92 & 0.25 & NA & 0.185 & NA & NA & $2 / 2$ \\
\hline V2 & 0.742 & 0.025 & 0.001 & 0.0004 & NA & 0.72 & 0.081 & NA & 0.185 & NA & NA & $2 / 3$ \\
\hline V6 & 0.641 & 0.28 & 0.001 & 0.003 & NA & 0.05 & 0.016 & NA & 0.67 & NA & NA & $2 / 3$ \\
\hline V1-trd & NA & 0.003 & 0.003 & 0.0002 & NA & 0.69 & 0.075 & NA & 0.037 & NA & NA & $3 / 4$ \\
\hline V2-trd & NA & 0.0008 & 0.004 & 0.0002 & NA & 0.41 & 0.019 & NA & 0.185 & NA & NA & $3 / 4$ \\
\hline V6-trd & NA & 0.05 & 0.0007 & 0.0004 & NA & 0.014 & 0.009 & NA & 0.77 & NA & NA & $2 / 5$ \\
\hline
\end{tabular}

Bonferroni correction to $\alpha=0.05$ is $\alpha=0.01667$ for three comparisons among platyrrhine groups, and $\alpha=0.0083$ for six comparisons among "prosimians". "sig" column gives number of comparisons significant at $P<0.005 / P<0.05$. All ANOVAs exhibited significant among group variance justifying post hoc follow-up. Variables ending in "-trd" were analyzed with a traditional ANOVA using the same species mean data. Traditional ANOVAs were only run for "prosimian" data, because $\Lambda$ was nonzero for these. All traditional ANOVAs again showed significant among group variance. Post hoc comparisons were carried out with Pairwise $t$-tests, and bonferroni corrected $P$ values apply again.

1993), we find the lowest P-value for the comparison between folivores and frugivores $P=0.0207$, though it is still not significant at the corrected critical value of $\alpha=0.005$ (or with a Dunn-Sidak approach). We would therefore qualify this statement and advise use of species mean data especially in cases where some taxa may be oversampled in number of specimens relative to others. We also recognize that unintentional or purposeful bias in taxonomic inclusion reflecting either phylogenetic, ecological, or body size considerations could magnify variation in the regression coefficients returned compared to what we observed in our study and do not recommend this either. Finally, if species mean data are being used and comprehensive taxonomic sampling is employed, it is not clear that PGLS methods are necessary in this context. It may be just as effective to limit regression computation to major clades (not necessarily monophyletic) where a relatively uniform scaling pattern can be observed (e.g., "prosimians" vs. haplorhines). Because the scaling relationship itself probably evolves, PGLS regressions applied to large clades may actually return less accurate results than ordinary regressions for some taxa. If only the intercept of the scaling relationship shifts, this is not too problematic; however, if behavioral or physiological changes also have caused a slope change then PGLS for all-incusive taxon samples may not be preferred. For instance, before concluding that there is a "fundamental allometry" to scaling of primate calcaneal proportions, Boyer et al. (2013) exhaustively compared slopes of scaling relationships for different subclades and showed them to be indistinguishable. With these caveats noted, SQ datasets computed from PGLS-derived equations represent the only other two datasets out of 14 evaluated where the post hoc comparison of folivores and frugivores is less than 0.05 (yet still much $>0.005$ ).

In sum, none of the variations between the original analytical protocol used by Winchester et al. (2014) and those used previously (e.g., Anthony and Kay, 1993) can be pointed to as explaining the lack of a frugivore/folivore signal in their data. Nonetheless, we are convinced from an overwhelming body of knowledge representing many independent studies that SQ should generally distinguish frugivorous from folivorous taxa.

This leads to the question of whether the lack of signal is driven by a particular clade. Our results mildly sup- port the notion that a lack of signal between platyrrhine folivores and frugivores drives the absence of signal among the combined sample, since the "prosimian" sample by itelf obtains significance for some methods of computing critical values for post hoc comparisons, while the platyrrhine sample does not. There are still other issues here including reduced power due to less taxonomic diversity and fewer folivorous taxa in the platyrrhine radiation than among "prosimians", and potential unreliability of Pagel's $K$ in the platyrrhine analysis. But there is little that can be done to ameliorate these problems.

This brings us to consideration of some of the variables we cannot evaluate directly at this time:

1. Winchester et al. (2014) used an unusual measurement protocol in that crest lengths follow the 3D surface of the tooth more closely than in most previous studies where crest lengths have been necessarily measured as straight lines using a reticle and microscope. Strait's (1991, 1993a,b, 2001) use of a reflex microscope generates data that should be fairly equivalent to that generated by Bunn et al. (2011) and Winchester et al. (2014), and she found good differentiation between her diet groups (though she did not include folivores in her datasets). This could be interpreted to show that surfacefollowing measurement protocols do not result in less ecologically salient measurements.

2. The study of Winchester et al. (2014) was the first study to assess the dietary signal in the platyrrhine $\mathrm{m} 2$. It is possible that this tooth is not as functionally informative as $\mathrm{m} 1$, the tooth used in other studies of platyrrhine SQ. Winchester et al. (2014) used m2 because they were interested in assessing the fidelity of the dietary signal in a homologous tooth position for the broadest phylogenetic sample possible ${ }^{2}$. This is a relevant goal since, for basal fossil taxa, there may be no objective criterion by which to select the first

${ }^{2}$ Kay (2004) showed that platyrrhine $\mathrm{m} 1$ values can be converted to SQs using coefficients from a strepsirrhine $\mathrm{m} 2$ regression to successfully reflect diet. However, it does not immediately follow that strepsirrhine and platyrrhine shearing values generated from the same strepsirrhine-based equation can be combined into a single dataset and expected to correlate with diet at that level. In fact, Boyer (2008) provides example computations suggesting that this approach would not be very useful relative to the goals of Winchester et al. (2014). 
molar for diet reconstruction over the second molar. In order to directly control for this last variable (tooth position) the authors of Winchester et al. (2014) would need to measure a dataset of mandibular first molars for the each of the platyrrhine specimens in their original dataset (ideally) and conduct the exact same series of analyses.

We have presented evidence here suggesting the platyrrhine $\mathrm{m} 2$ shearing length has a weaker dietary signal than the $\mathrm{m} 2$ shearing lengths of other primates. We speculate that $\mathrm{m} 2$ shearing length of platyrrhines also has a weaker dietary signal than the platyrrhine m1. It would appear that this is the primary reason for the unexpected results of Winchester et al. (2014) regarding a lack of differentiation between folivores and frugivores in SQ. Though analyses conducted in this study point toward this explanation, direct comparison of $\mathrm{m} 1$ and m2 platyrrhine shearing datasets are needed to further test it. This hypothesis also predicts that the topographic variables [Relief Index (Boyer, 2008) and Dirichlet Normal Energy (Bunn et al. 2011)] computed for platyrrhine m2's by Winchester et al. (2014) should exhibit greater differentiation between folivores and frugivores when computed on platyrrhine m1's [as these variables already significantly distinguished folivore and frugivore m2's in Winchester et al.'s (2014) analyses].

\section{SUMMARY AND CONCLUSION}

In contrast to an expectation established by previous studies of SQ on lower molars, Winchester et al. (2014) failed to recover a significant difference between $S Q$ in a sample of folivores and frugivores representing platyrrhines and "prosimians". A number of errors and methodological differences between their approach and more traditional SQ computations (e.g., Anthony and Kay, 1993) are noted. Correction of errors followed by systematic modification of protocol and re-analysis of the Winchester et al. (2014) dataset still fails to recover differences between folivores and frugivores. However, removing the postentocristid crest length from the computation of overall shearing crest length does generally improve the distinction among different dietary groups (though not for platyrrhines considered in isolation of "prosimians"). Finally, phylogenetic ANOVA run separately on platyrrhine and prosimian groups suggests that platyrrhines exhibit less differentiation than "prosimians" in $\mathrm{m} 2$ shearing when comparing folivores and frugivores. We therefore speculate that the most likely explanation for the surprising lack of distinctiveness between platyrrhine folivores and frugivores in Winchester et al.'s (2014) dataset is that the authors used the $\mathrm{m} 2$ for their analyses instead of the $\mathrm{m} 1$ as in other studies of platyrrhine SQ. This helps justify the assumption of previous studies that the functional demands on the platyrrhine $\mathrm{m} 2$ are not as consistent as those on the $\mathrm{m} 1$ in contrast to non-platyrrhines where the $\mathrm{m} 2$ has been the tooth of choice. Going forward, in order to study evolution of dietary signal in the primate dentition in a more comprehensive way, taxonomically comprehensive datasets including shearing and shape measures for both $\mathrm{m} 1$ and $\mathrm{m} 2$ should be sought. Not only will this allow a more robust model for diet estimation for all clades included, it could provide a context for answering questions about when in primate evolutionary history, in which clades, and in association with what other morphological and environmental changes the main target of selection for improved shearing potential shifted from the $\mathrm{m} 2$ to the $\mathrm{m} 1$.

\section{ACKNOWLEDGMENTS}

The authors acknowledge the staff and curators at the American Museum of Natural History Mammalogy Dept. (Darin Lunde, Eileen Westwig, Neil Duncan, Nancy Simmons). Additional collections staff to whom we are indebted include those at the Smithsonian (Linda Gordon and Darin Lunde) and Harvard Museum of Comparative Zoology. The curators of the Stony Brook University Anatomy Department's collection must also be thanked for access. We thank Siobhan Cooke for access to Brachyteles specimens. Jukka Jernvall provided access to Prolemur simus specimens for molding. We thank Stefan Judex and Clint Rubin of the Stony Brook University Center for Biotechnology for access to their scanners at the time of original data collection.

\section{LITERATURE CITED}

Allen KL, Cooke SB, Gonzales L, Kay, RF. in review. Dietary inference from upper and lower molar morphology in platyrrhine primates. PLoS One.

Anthony MRL, Kay RF. 1993. Tooth form and diet in ateline and alouattine primates: reflections on the comparative method. Am J Sci 293A:356-382.

Arnold C, Matthews LJ, Nunn CL. 2010. The 10kTrees Website: a new online resource for primate phylogeny. Evol Anthropol 19:114-118.

Blomberg SP, Garland T, Ives AR. 2003. Testing for phylogenetic signal in comparative data: behavioral traits are more labile. Evolution 57:717-745.

Boyer DM. 2008. Relief index of second mandibular molars is a correlate of diet among prosimian primates and other euarchontan mammals. J Hum Evol 55:1118-1137.

Boyer DM, Seiffert ER, Gladman JT, Bloch JI. 2013. Evolution and allometry of calcaneal elongation in living and extinct primates. PLoS One 8:e67792.

Bunn JM, Boyer DM, Jernvall J, Lipman Y, Daubechies I. 2011. Dirichlet normal surface energy of tooth crowns, a new technique of molar shape quantification for dietary inference, compared with previous methods in isolation and in combination. Am J Phys Anthropol 145:247-261.

Campbell SE, Cuozzo FP, Sauther ML, Sponheimer M, Ferguson VL. 2012. Nanoindentation of lemur enamel: an ecological investigation of mechanical property variations within and between sympatric species. Am J Phys Anthropol 148:178-190.

Cuozzo FP, Sauther ML. 2006. Severe wear and tooth loss in wild ring-tailed lemurs (Lemur catta): a function of feeding ecology, dental structure, and individual life history. J Hum Evol 51:490-505.

Cuozzo FP, Sauther ML. 2012. The dental ecology of ring-tailed lemurs (Lemur catta). In: Masters JC, Gamba M, Génin F, editors. Leaping ahead: advances in prosimian biology. New York: Springer. p 157-163.

Hiiemae KM, Kay RF. 1973. Evolutionary trends in dynamics of primate mastication. In: Montagna W, editor. Symposia of the 4th International Congress of Primatology, Vol. 3: Craniofacial Biology of Primates: Karger, Basel. p 28-64.

Kay R, Hiiemae K. 1974. Jaw movement and tooth use in recent and fossil primates. Am J Phys Anthropol 40:227-256.

Kay RF. 1975. The functional adaptations of primate molar teeth. Am J Phys Anthropol 43:195-216.

Kay RF. 1977. The evolution of molar occlusion in Cercopithecidae and early catarrhines. Am J Phys Anthropol 46:327352 . 
Kay RF. 1978. Molar structure and diet in extant Cercopithecidae. In: Butler P, editor. Development, function, and evolution of teeth. London: Academic Press. p 309-339.

Kay RF. 2004. A synopsis of the phylogeny and paleobiology of Amphipithecidae, South Asian middle and late Eocene primates. Anthropol Sci (Jpn) 1-12.

Kay RF, Cartmill M. 1977. Cranial morphology and adaptations of Palaechthon nacimienti and other Paromomyidae (Plesiadapoidea, ? Primates), with a description of a new genus and species. J Hum Evol 6:19-35.

Kay RF, Sussman RW, Tattersall I. 1978. Dietary and dental variations in the genus Lemur, with comments concerning dietary-dental correlations among Malagasy primates. Am J Phys Anthropol 49:119-128.

King SJ, Arrigo-Nelson SJ, Pochron ST, Semprebon GM, Godrey LR, Wright PC, Jernvall J. 2005. Dental senescence in a longlived primate links infant survival to rainfall. Proc Natl Acad Sci USA 102:16579-16583.

Kirk EC, Simons EL. 2001. Diets of fossil primates from the Fayum depression of Egypt: a quantitative analysis of molar shearing. J Hum Evol 40:203-229.

Lanyon JM, Sanson GD. 1986. Koala (Phascolarctos cinereus) dentition and nutrition. II. Implications of tooth wear in nutrition. J Zool Lond Ser A 209:169-181.

Millette JB, Sauther ML, Cuozzo FP. 2009. Behavioral responses to tooth loss in wild ring-tailed lemurs (Lemur catta) at the Beza Mahafaly Special Reserve, Madagascar. Am J Phys Anthropol 140:120-134.

Sauther ML, Cuozzo FP. 2009. The impact of fallback foods on wild ring-tailed lemur biology: a comparison of intact and anthropogenically disturbed habitat. Am J Phys Anthropol 140:671-686.

Sheine WS, Kay RF. 1977. An analysis of chewed food particle size and its relationship to molar structure in primates Cheirogaleus medius and Galago senegalensis and insectivoran Tupaia glis. Am J Phys Anthropol 47:15-20.

Sheine WS, Kay RF. 1982. A model for comparison of masticatory effectiveness in primates. J Morph 172:139-149.

Strait SG. 1991. Dietary Reconstruction in Small-Bodied Fossil Primates [Ph.D. Dissertation]. Stony Brook, NY: State University of New York at Stony Brook.

Strait SG. 1993a. DIfferences in occlusal morphology and molar size in frugivores and faunivores. J Hum Evol 25:471-484.

Strait SG. 1993b. Molar morphology and food texture among small-bodied insectivorous mammals. J Mammal 74:391402.

Strait SG. 2001. Dietary reconstruction of small-bodied omomyoid primates. J Vertebr Paleontol 21:322-334.

Winchester JM, Boyer DM, Cooke SE, St.Clair EM, Ledogar JA Gosselin-Ildari AD. 2014. Dental topography of platyrrhines and prosimians: convergence and contrasts. Am J Phys Anthropol 153:29-44. 